\title{
Floating Power Station (Mars the Future Wind-Mill)
}

\author{
${ }^{1}$ P.YASHWANTH, ${ }^{2}$ P.VENKATA GANESH, ${ }^{3}$ E.ARUNPRAKASH, ${ }^{4}$ S.BENISHA \\ 1, 2 III-EEE, Narasu's Sarathy Institute of Technology, \\ ${ }^{3,4}$ Assistant Professor, Narasu's Sarathy Institute of Technology, Salem.
}

\begin{abstract}
In this paper a simplified approach of Magenn Air Rotor System (MARS) power which is one of the recently found energy source. MARS is a lighter wind turbine that rotates about a horizontal axis in response to wind, generating electrical energy. This electrical energy is transferred down for immediate use, or to a set of batteries for later use, or to the power grid.Helium (an inert non-reactive lighter than air) sustains the Air Rotor which ascend to an altitude for best wind and its rotation also causes the Magnus effect. This provides additional lift, keeps the device stabilized, and keeps it positioned within a very controlled and restricted location. This is the latest technology in Energy sector and cheaper than other techniques and Eco friendly.
\end{abstract}

\section{Introduction:}

The 'whirling' noise of windmills is unpleasant to some people, more so if you live close to one. However, harnessing wind power is important, so a number of alternatives to a generic windmill have been proposed. An Ontario-based company is working to place "turbines" floating hundreds of feet high above the ground now let us see the working principle of "MARS" Magenn Power Air Rotor System.

The "Magenn Power Air Rotor System" (MARS) is a helium filled, cylindrical device, deployed at $1000 \mathrm{ft}$ maximum altitude, which rotates about its horizontal axis in response to the wind, generating clean energy at a low cost than competing systems.

\section{How The Mars Turbine Works:}

In a few years, you may see scores of giant blimps floating overhead. Those blimps are actually wind turbines. They're not your typical turbine, though. These extremely mobile energy generators, christened with the acronym MARS, will float high the air at altitudes ranging from 600 to 1,000 feet (183 to 305 meters).

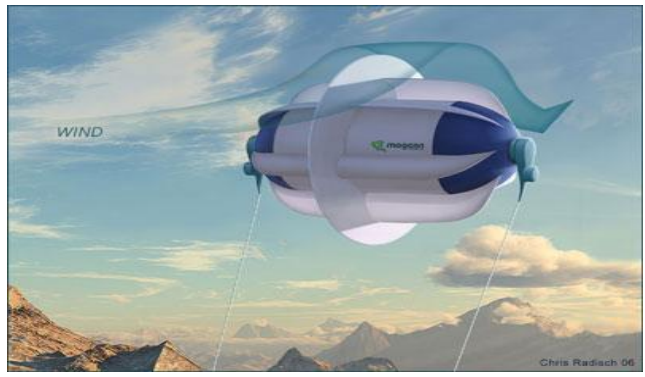

Fig: Mars Structure.

While the current 100,000 megawatts of electricity generated worldwide by wind is impressive, it only accounts for a small percentage of the world's total electricity production.

At the most basic level, generating electricity from the movement of wind is straightforward. Which, in turn, cause an attached generator to also spin. The generator then converts that moving energy of the wind into electricity using electromagnetic induction, which involves using the opposite charges of a magnet to create an electric current.

Instead of the large pinwheel blades that are typical of wind turbines though, the blades of the MARS turbine are actually part of the three-dimensional blimp itself. The blades catch the wind, causing the entire blimp to spin around. After the generator converts that movement into electricity, it's transferred down the turbine's long tether.

Although the basic concept behind it is the same as that behind traditional wind turbines -- the conversion of one form of energy (wind energy) into another (electricity) the MARS turbine possesses several differences that could make it appeal to a wider market. One of those differences is that it doesn't rely on a large tower to hold it up. 


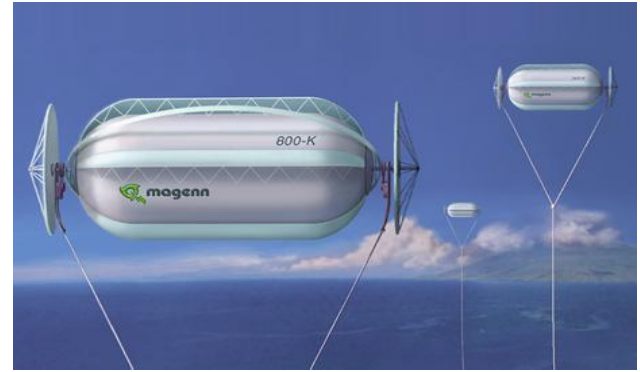

Fig: Mars Structure

\section{Inside Mars}

Magenn Power designed its turbine not only for easy deployment, but also for easy maintenance. Obviously, a blimp-like object floating at 1,000 feet (305 meters) could receive quite a beating from the elements, but the company estimates the MARS should last at least 15 years before requiring maintenance. The woven outer part is actually made from the same material used in bulletproof vests and is lined with a coating that protects it from UV rays and abrasion. The inner portion is coated with Mylar to prevent the helium gas from escaping.

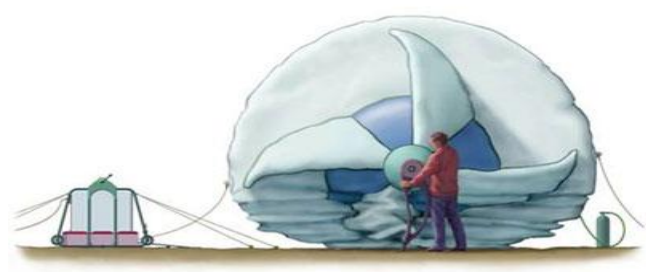

Fig: Helium Filled Mars

MARS envelope will be made of a durable material like that used in bullet proof vests. Since the MARS is located at such high altitudes, it was also designed to be able to withstand strong winds. While conventional turbines will shut down at wind speeds in excess of $45 \mathrm{mph}$, the MARS can function at speeds greater than $63 \mathrm{mph}$. At the other end of the spectrum, the MARS turbine can also convert wind energy into electricity.

Part of what enables the MARS to stay vertical at high wind speeds is due to something called the Magnus effect.When the object spins, an area of high pressure forms beneath it and causes it to rise. Since the effect increases as wind speed increases, the MARS is able to use it in combination with the lift from the helium to maintain a near vertical position and not lean in high winds.

The wide range of speeds at which it can operate means that the MARS can deliver output much closer to its rated capacity than standard designs can.

IV. How Does It Works

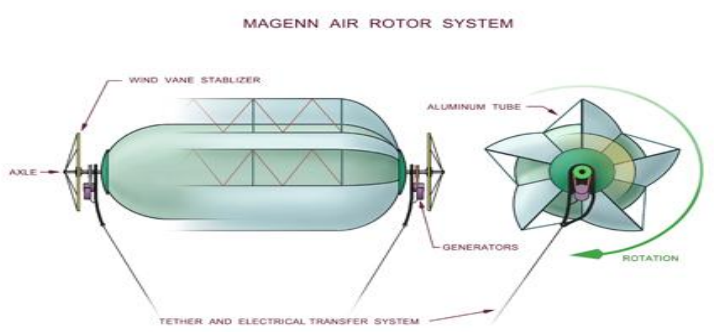

The Magenn Power Air Rotor System (MARS) is an innovative lighter-than-air tethered device that rotates about a horizontal axis in response to wind, efficiently generating clean renewable electrical energy at a lower cost than all competing systems. This electrical energy is transferred down the tether to a transformer at a ground station and then transferred to the electricity power grid. Helium (an inert non-reactive lighter than air gas) sustains the Air Rotor which ascends to an altitude for best winds and its rotation also causes the Magnus effect. This provides additional lift, keeps the device stabilized, keeps it positioned within a very controlled and restricted location, and causes it to pull up overhead rather than drift downwind on its tether. 


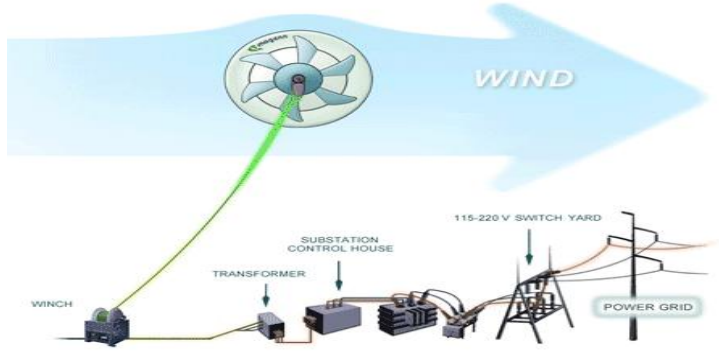

Fig: Working Of Mars.

The cylindrical MARS unit is filled with helium, which provides the lift necessary to keep it in the air, additional lift is provided by the Magnus effect, where a rotating object in the air can also generate lift for itself. This effect also enables the unit to stay in place, rather than constantly drift downwind. Once the wind passes over the unit, electricity is generated by rotation of the MARS unit, and it is then transferred by cables to the ground into a transformer.

All competing wind generators use bladed two-dimensional disk-like structures and rigid towers. The Magenn Power Air Rotor system is a closed three-dimensional structure (cylinder). It offers high torque, low starting speeds, and superior overall efficiency thanks to its ability to deploy higher. The closed structure allows Magenn Power to produce wind rotors from very small to very large sizes at a fraction of the cost of current wind generators.

\section{Advantages}

Air turbines have numerous advantages, they are highly flexible deployment, have lower costs, have better efficiency than competing systems, and are more environmentally friendly.

Wind farms can be placed closer to demand centers, reducing transmission line costs and transmission line loses.

In terms of electrical energy output, the Magenn systems are less expensive than competing wind power systems (under $15 \mathrm{c}$ per $\mathrm{kWh}$ ).air turbines can be placed close to wherever they are needed, Air turbines can operate at higher wind speeds than conventional wind turbines Conventional wind generators are only operable in wind speeds between 3 meters/sec and 28 meters/sec. Magenn Air Rotors are operable between 1 meter/sec and in excess of 28 meters/sec.

- Air turbines float high up in the air, up to $1000 \mathrm{ft}$, thus capitalizing on higher wind speeds. Visual impact is also reduced, as air turbines are less visible than conventional wind turbines. Noise impact is also reduced due to the high altitude operation of the air turbine is less of an impact on the bird and bats population. Air turbines are softer objects, without any deadly solid blades.

- Air turbines are highly mobile, and can be moved easily. This is especially good for emergency disaster relief situations.

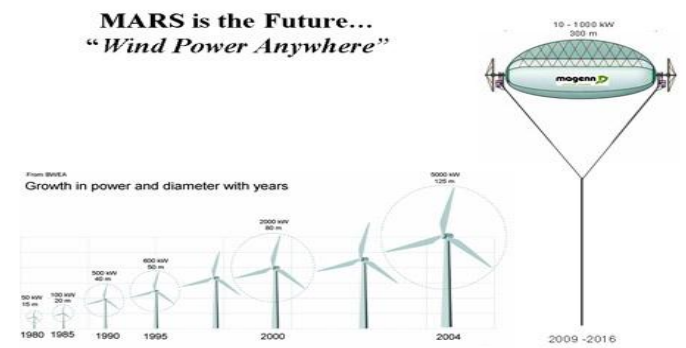

\section{APPLICATIONS}

MAGENN technology will be applied off-grid and combined with diesel power for developing nations, island nations, farms, remote areas, cell towers, exploration equipment, oil and gas wells, mining sites, offshore drilling stations, and backup power \& water pumps. MAGENN rotors could also be used for on-grid applications for farms, factories, and remote communities. 


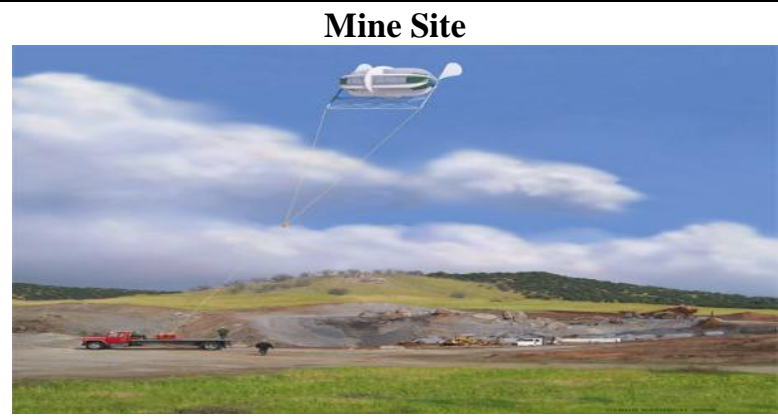

\section{Oil Rig}

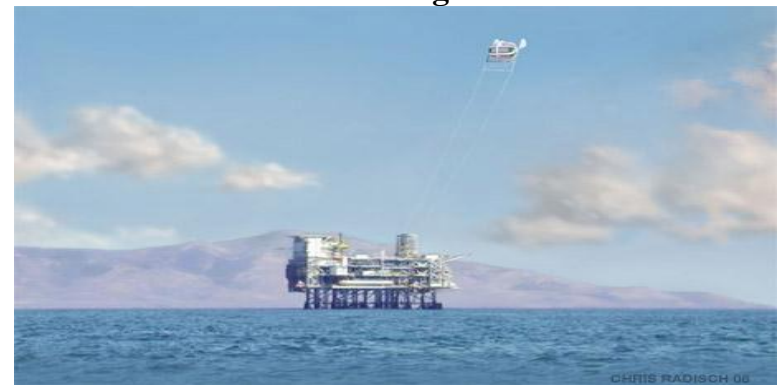

Future Magenn Air Rotor System (MARS)

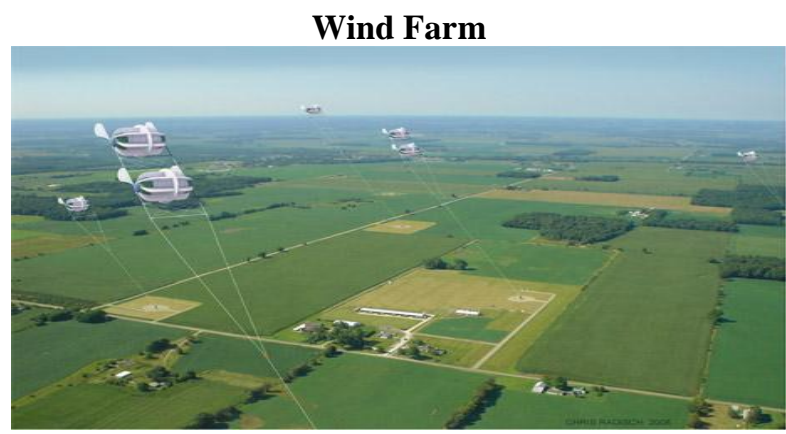

Remote Exploration

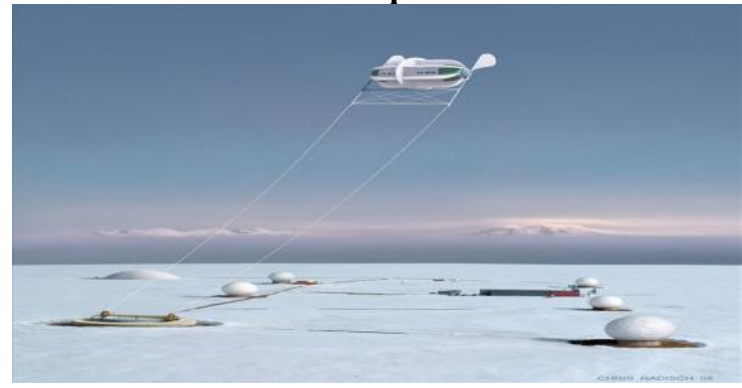

VI. Conclusion

We know that Wind energy is a CLEAN Energy i.e. Pollution-free and eco-friendly. Also wind energy is a renewable energy. Our M.A.R.S is a hot-new mobile power generating system uses the wind energy in the effective manner so as to generate more electrical power. This system proves as better \& more efficient power generating system than the present wind power systems. These kinds of newer technologies are necessary for compensating the increase in present world power consumption in effective manner. Let us use these kinds of cost \& eco-friendly system for power generation.

\section{References}

[1]. Alter,Lloyd."MagennAirRotorSystemFinallyFloats."Treehugger.com.May5,2008.(July7, 2008).http://www.treehugger.com/files/2008/05/magenn-air-rotor-floats.php

[2]. American Wind Energy Association. "awea.org." 2008. (July 7, 2008). http://www.awea.org/

[3]. Layton,Julia."HowWindPowerWorks."HowStuffWorks.2008.(July3,2008).http://science.howstuffworks.com/wind-power.htm 